\title{
Some Results And A Conjecture For Manna's Stochastic Sandpile Model
}

\author{
Deepak Dhar \\ Department of Theoretical Physics, \\ Tata Institute of Fundamental Research, \\ Homi Bhabha Road, Mumbai 400 005, INDIA
}

\begin{abstract}
$\underline{\text { Abstract }}$
We present some analytical results for the stochastic sandpile model studied earlier by Manna. In this model, the operators corresponding to particle addition at different sites commute. The eigenvalues of operators satisfy a system of coupled polynomial equations. For an $L \times L$ square, we construct a nontrivial toppling invariant, and hence a ladder operator which acting on eigenvectors of the evolution operator gives new eigenvectors with different eigenvalues. For periodic boundary conditions in one direction, one more toppling invariant can be constructed. We show that there are many forbidden subconfigurations, and only an exponentially small fraction of all stable configurations are recurrent. We obtain rigorous lower and upper bounds for the minimum number of particles in a recurrent configuration, and conjecture a formula for its exact value for finite-size rectangles.
\end{abstract}

PACS nos: 05.20.Ln, 02.50.Ga, 02.10.Pk, 81.05.Rm

\section{Introduction}

Many cellular automaton 'sandpile' models have been studied in recent years as simple theoretical models of self-organized criticality. The prototype of these models is the Abelian sandpile model (ASM), proposed by Bak et al in 1987 [1, 2]. Many properties of this model can be determined analytically using the fact that the operators corresponding particle addition in this model generate an abelian group, and the model is equivalent to the $q \rightarrow 0$ limit of the Potts model [3]. Several variations of the ASM have been studied in the past with a view to understand the parameters that determine the different universality classes of self-organized critical behavior 1 , 5, 6. These include models in which particle transfer is directed [7], or models in which the toppling condition or the number of sandgrains transferred depends on the local slope rather than local height. In this respect, it has been realized that stochasticity in toppling rules can lead to different critical behavior than models with deterministic toppling rules [8, 9, 10].

As a physical motivation for the study of such models, we note that stochasticity in the toppling rules may be taken as a simple phenomenological attempt to take into account in a theoretical description the variation in shape and smoothness of different grains in real 
granular media. It has been argued that a stochastic one-dimensional sandpile model seems to describe well the behavior of experimental results on avalanches in ricepiles [1].

In this paper, we study a class of stochastic sandpile models, the prototype of which is the so-called Manna model [8]. It was recently proved that this model also shows the abelian property, and is a special case of the more general Abelian Distibuted Processors (ADP) model [3]. In the following, we shall use the terms deterministic ASM (DASM) and stochastic ASM (SASM) if we need to distinguish between these classes of models. We will show that some of the analytical techniques of DASM are also useful in studying the SASM.

The plan of this paper is as follows: In section 2, we recapitulate the definition of the Manna model. We study this model in two dimensions on a square lattice, but most of the treatment can be easily generalized to higher dimensions. In section 3, we describe the abelian ring structure of the algebra generated by the particle addition operators. The simultaneous eigenvalues of these operators satisfy a system of coupled quadratic equations. In section 4, we show that for the case of an $L \times M$ rectangle, if $L=M$, there is a nontrivial toppling invariant. We use this to construct a ladder operator that gives $(L+1)$ simultaneous eigenvectors of the particle addition operators from one such eigenvector. For periodic boundary conditions in the y-direction, and $M=L+1$, we can construct one more toppling invariant, and this raises the number of eigenvectors generated from one vector to $M^{2}$. In section 5, we prove the existence of forbidden sub-configurations (FSC's) in the model. We describe an algorithm to construct the set of minimal FSC's. In section 6, we study the recurrent configurations of the model which have minimum number of sandgrains. We prove upper and lower bounds on this number for a finite rectangle, and conjecture that the upper bound always coincides with the exact value.

\section{Definition of the Model}

We consider a two-dimensional square lattice. The sites of the lattice will be labelled by their integer Euclidean coordinates $(x, y), \quad 1 \leq x \leq L, 1 \leq y \leq M$. At each site $(x, y)$, there is a non-negative integer $z_{x, y}$ called the height of the pile. In addition, at each site,we assume that we have a pseudo-random-number generator (PRNG), which gives a number lying between 0 and 1 on each request using a deterministic algorithm. The $n$-th output from a PRNG depends only on its initial setting (seed), and $n$, and not in any way on the evolution of the system, or status of other PRNG's. Equivalently, we can think of the PRNG's as an infinite stack of independent random numbers at each site.

If the height at any site does not exceed 1, the configuration is said to be stable. If the system is in a stable configuration, we choose a site at random and add a grain of sand there. as a result of the addition, height at that site increases by 1 . If this makes the site unstable, we relax the system using the relaxation rule given below. Once all unstable sites are relaxed, we add another particle. We shall assume that the probability of addition is nonzero for all sites. It follows from the general theory of ASM's that the steady state of the system is then independent of the precise choice of these probabilities.

The relaxation rule is as follows: Any site whose height exceeds 1, relaxes by toppling, and throwing two grains out. Where these grains go is decided by drawing a new random number from the local PRNG. In terms of stacks, we pop up a number from the local stack. 
If the number is less that $1 / 2$, one particle is transferred to each of the two horizontal neighbors of the site. Else, one particle is transferred to each of the two vertical neighbors. If the transfer renders some other sites unstable, these are toppled in turn, until a stable configuration is reached. Sandgrains can leave the system if a toppling occurs at a boundary site.

\section{The Abelian Ring of Operators}

This model as a special case of the more general Abelian Distributed Processors Model (ADP) [3], and the abelian character of the model becomes obvious. We define particle addition operators $\mathbf{A}_{x, y}$ which acting on a stable configurations $\mathcal{C}$, gives the stable configuration obtained after adding a particle at $(x, y)$, and relaxing the system. Here the stable configurations are specified by the heights $\left\{z_{x, y}\right\}$, and the state of the PRNG at each site (equivalently, a stack of random numbers at each site). It is easy to see that if we start with a configuration with two or more unstable sites, we get the same configuration, independent of the order in which the unstable sites are relaxed. Using this property repeatedly, it follows that

$$
\left[\mathbf{A}_{x, y}, \mathbf{A}_{x^{\prime}, y^{\prime}}\right]=0 \text {, for all sites }(x, y) \text { and }\left(x^{\prime}, y^{\prime}\right) \text {. }
$$

We may now assume that the internal state of these PRNG's as inaccessible to the outside observer, and that there are no observable correlations between different outputs of the PRNG's ( i.e. they are 'good' PRNG's). Then the deterministic evolution of avalanches in the (sandpile heights+ PRNGs) system may be described equally well as a Markovian relaxation of the subsystem described in terms of sandpile heights alone.

Given a particular state of the PRNG's at each site, addition of two particles at different sites gives same final stable configuration, independent of the order of topplings. A different state of PRNG's can lead a different final state, but still independent of order of topplings. If we do not know the state of the PRNG's, we have to average over their different possible states. Then, the final state is not fixed, only the probabilities of different outcomes can be given.

In Manna's original formulation of the model, the transfer direction was decided randomly at each toppling. The advantage of the specific implementation used here with a different PRNG at each site makes the fact that abelian character of the model obvious. The abelian property, once established, is clearly independent of the details of implementation of the algorithm. So, one could as well use the same PRNG to decide the direction of particle transfer in topplings at different sites.

We consider the $2^{L M}$-dimensional vector space $\mathcal{V}$, whose basis vectors $|\mathcal{Z}\rangle$ are labelled by the stable height configurations $\mathcal{Z} \equiv\left\{z_{x, y}\right\}$. If the probability that at time $t$ the config-

uration of the sandpile is $\mathcal{Z}$ is denoted by $\operatorname{Prob}(\mathcal{Z}, t)$, then the state of the system can be represented by a vector $\mid P(t)>\in \mathcal{V}$

$$
\left|P(t)>=\sum_{\mathcal{Z}} \operatorname{Prob}(\mathcal{Z}, t)\right| \mathcal{Z}>
$$


We define particle addition operators $\mathbf{a}_{x, y}$ as linear operators acting on $\mathcal{V}$ as follows: for all configurations $\mathcal{Z}, \mathbf{a}_{x, y} \mid \mathcal{Z}>$ gives the probability vector of the system obtained by taking the system in the stable configuration $\mathcal{Z}$, adding a particle at site $(x, y)$, and relaxing the system until a stable configuration is reached. For stochastic toppling rules, the resulting state is not necessarily a basis vector corresponding to a unique configuration, but a linear combination of different basis vectors. Note that action of any of these operators on a given configuration gives a unique probability state vector, and action of these $\left\{\mathbf{a}_{x, y}\right\}$ 's is deterministic, in the same way as evolution of the wave-function in quantum mechanics is deterministic. The Abelian property of the Manna model means that the probabilities of different final stable configurations are independent of the order of topplings and particle additions.

The commutativity of the operators $\{\mathbf{A}(\mathbf{x}, \mathbf{y})\}$ implies that the operators $\left\{\mathbf{a}_{x, y}\right\}$ also commute with each other:

$$
\left[\mathbf{a}_{x, y}, \mathbf{a}_{x^{\prime}, y^{\prime}}\right]=0, \text { for all sites }(x, y) \text { and }\left(x^{\prime}, y^{\prime}\right) \text {. }
$$

This abelian property simplifies the analysis of the model considerably. There are additional relations satisfied by the operators $\left\{\mathbf{a}_{x, y}\right\}$. Adding two particles at a site will cause it to topple, whatever the initial configuration, and then with equal probability two particles are transferred either horizontally, or vertically. This implies that the operators $\left\{\mathbf{a}_{x, y}\right\}$ satisfy the equations

$$
\mathbf{a}_{x, y}^{2}=\frac{1}{2}\left(\mathbf{a}_{x-1, y} \mathbf{a}_{x+1, y}+\mathbf{a}_{x, y-1} \mathbf{a}_{x, y+1}\right), \text { for all } x, y,(1 \leq x \leq L, 1 \leq y \leq M) .
$$

In writing these equations, we have assumed the boundary conditions

$$
\begin{aligned}
& \mathbf{a}_{0, y}=\mathbf{a}_{L+1, y}=1, \text { for } 1 \leq y \leq M \\
& \mathbf{a}_{x, 0}=\mathbf{a}_{x, M+1}=1, \text { for } 1 \leq x \leq L
\end{aligned}
$$

If we assume periodic boundary conditions in the y-direction, the Eqs.(6) are replaced by the equations

$$
\mathbf{a}_{x, y}=\mathbf{a}_{x, y+M}, \text { for all } x, 1 \leq x \leq L \text {, all } y .
$$

Any vector $\mathbf{v} \in \mathcal{V}$ can be expressed as $P(\mathbf{v}) \mid \Phi>$, where $P(\mathbf{v})$ is a polynomial in $\left\{\mathbf{a}_{x, y}\right\}$, with the highest power of any $\mathbf{a}_{x, y}$ being 1 , and $\mid \Phi>$ is the vector corresponding to configuration with all sites empty. We can multiply two such polynomials $P_{1}$ and $P_{2}$, and reduce the highest power of operators $\mathbf{a}_{x, y}$ using the reduction rules Eq.(4).

We may think of the operators $\left\{\mathbf{a}_{x, y}\right\}$ as abstract symbols, and study the algebra of polynomials in these variables defined by the usual addition, and multiplication. Using the reduction rules (4), the maximum degree of any symbol in the polynomials can be reduced to 1 . Clearly, the set of all such polynomials forms a commutative ring.

It is instructive to compare this situation with that of the DASM. In the latter case also, there are reduction rules which reduce large powers of abelian operators $\left\{\mathbf{a}_{x, y}\right\}$ to lower ones, but the expression on the right-hand side of Eq. (4) consists of a single term. Then the operators define a finite-dimensional abelian semi-group, and if we restrict to the set of 
recurrent states, we get an abelian group with an well-defined multiplicative inverse [3]. In the present case, the reduction rules give us not a single term, but a linear combination of terms. To form an algebra closed under addition and multiplication, we have to include all linear combinations of terms. Thus, the number of elements of the algebra is infinite.

The operators $\left\{\mathbf{a}_{x, y}\right\}$ have an obvious representation as $2^{L M} \times 2^{L M}$ matrices (defined by their action on basis vectors of $\mathcal{V}$ ). These matrices are in general not diagonalizable, only reducible to the Jordan canonical form, and there may not be eigenvectors corresponding to each repeated eigenvalue. For the DASM, all transient states correspond to the zero eigenvalue of the operators $\left\{\mathbf{a}_{x, y}\right\}$, and all other eigenstates are of modulus 1. For the SASM, there may be a set of configurations such that if the system is any one of them, with a finite probability, it makes a transition within the same set, and with a finite probability it gets out. If the system can never get back to these states, these states are transient, but there need not be any zero eigenvalue of the operators $\left\{\mathbf{a}_{x, y}\right\}$ corresponding to such transient states. Also, amongst the recurrent states, one can have two different initial probability vectors that yield the same resultant vector. Thus, there can be eigenvectors of zero eigenvalue in the recurrent sector, and in general, the inverse operator $\mathbf{a}_{x, y}^{-1}$ need not exist, even if we restrict ourselves to the set of recurrent configurations of the model.

Consider one of the operators, say $\mathbf{a}_{1,1}$. Let $a_{1,1}$ be one of the eigenvalues of $\mathbf{a}_{1,1}$. Let $\mathcal{V}_{1}$ be the subspace of $\mathcal{V}$ spanned by the (right) eigenvectors of $\mathbf{a}_{1,1}$ corresponding to the eigenvalue $a_{1,1}$. There is at least one such eigenvector, so $\mathcal{V}_{1}$ is non-null. We pick one of the other addition operators, say $\mathbf{a}_{1,2}$. From the fact that $\mathbf{a}_{1,2}$ commutes with $\mathbf{a}_{1,1}$, it immediately follows that $\mathbf{a}_{1,2}$ acting on any vector in the subspace $\mathcal{V}_{1}$ leaves it within the same subspace. Diagonalizing $\mathbf{a}_{1,2}$ within this subspace, we construct a possibly smaller, but still non-null, subspace $\mathcal{V}_{2}$ which is spanned by simultaneous eigenvectors of $\mathbf{a}_{1,1}$ and $\mathbf{a}_{1,2}$ with eigenvalues $a_{1,1}$ and $a_{1,2}$. Repeating this argument with other operators, we can construct vectors which are simultaneous eigenvectors of all the $\left\{\mathbf{a}_{x, y}\right\}$. Let $\mid \Psi>$ be such a vector, with

$$
\mathbf{a}_{x, y}\left|\Psi>=a_{x, y}\right| \Psi>\text {, for all }(x, y) .
$$

Then the eigenvalues $\left\{a_{x, y}\right\}$ satisfy the coupled set of quadratic equations

$$
a_{x, y}^{2}=\frac{1}{2}\left(a_{x-1, y} a_{x+1, y}+a_{x, y-1} a_{x, y+1}\right), \text { for all } x, y ; \quad 1 \leq x \leq L, 1 \leq y \leq M .
$$

These are $L M$ quadratic equations in the $L M$ complex variables $\left\{a_{x, y}\right\}$. In general, they have $2^{L M}$ solutions. It seems difficult to write down the general solution of these equations explicitly. However, it is easy to see that all $a_{x, y}=1$ is certainly a solution of these equations. The corresponding eigenvector $\mid \Psi>$ gives the steady state. Unlike the DASM case, here we are not able to write down explicitly the probabilities of different recurrent configurations in the steady state.

\section{Toppling Invariants}

Toppling invariants have been found to be very useful in characterizing the structure of recurrent states in the DASM case [12]. Consider a DASM on N sites, whose configuration is 
specified by the heights $\left\{z_{i}\right\}, i=1,2 \ldots N$, and the toppling matrix is $\Delta$. For any arbitrary configuration $\mathcal{Z} \equiv\left\{z_{i}\right\}$, a toppling invariant $I$ is defined as a integer function linear in the height variables by the equation

$$
I(\mathcal{Z})=\sum_{i} g_{i} z_{i}, \quad(\bmod d)
$$

where $d$ is some integer, and $g_{i}$ are integers chosen so that $I$ does not change under toppling at any of the sites $j$. The $N$ different choices of $j$ give $N$ conditions to be satisfied by the as yet undetermined coefficients $\left\{g_{i}\right\}$. For suitable choice of $d$, these equations have a non-trivial solution, and in fact one can find a minimal set of toppling invariants in terms of the Smith normal form of the matrix $\Delta[12$.

For the present model, we define a toppling invariant $I_{1}$ by the equation

$$
I_{1}(\mathcal{Z})=\sum_{x, y} g(x, y) z_{x, y}, \quad(\bmod d)
$$

For this to be unchanged if there is a toppling at $(x, y)$, we must have

$$
2 g(x, y)=g(x-1, y)+g(x+1, y), \quad(\bmod d) .
$$

and

$$
2 g(x, y)=g(x, y-1)+g(x, y+1), \quad(\bmod d) .
$$

These are $2 L M$ equations for the $L M+1$ unknown coefficients $g(x, y)$ and $d$. In general, we would not expect any nontrivial solution. Interestingly, for the special case $L=M$, these equations have a simple nontrivial solution for $d=L+1$ :

$$
g(x, y)=x y
$$

We show below that toppling invariants can also be used as ladder operators, which acting on an eigenvector of the evolution operator, gives rise to another eigenvector of the same with a different eigenvalue. For the DASM, this is not very important, as the evolution operator can be diagonalized directly, and all the eigenvectors are easily determined. For the SASM, the eigenvectors are not easily determined, and the fact that toppling invariants can be used to construct ladder operators provides useful information.

We define an operator $\mathbf{S}$, diagonal in the configuration basis by the equation

$$
\mathbf{S}=\exp \left(\frac{-2 \pi i I_{1}}{L+1}\right)
$$

Clearly, $\mathbf{S}$ is also invariant under toppling. Simple algebra then gives

$$
\mathbf{S}^{-1} \mathbf{a}_{x, y} \mathbf{S}=\exp \left(\frac{2 \pi i x y}{L+1}\right) \mathbf{a}_{x, y}
$$

Let $\mid \Psi>$ is a simultaneous eigenvector of the operators $\left\{\mathbf{a}_{x, y}\right\}$, with eigenvalues $\left\{a_{x, y}\right\}$, $1 \leq x \leq L, 1 \leq y \leq M$. Then, it follows that $\mathbf{S} \mid \Psi>$ is also a simultaneous eigenvector of all these operators with eigenvalues $\left\{\tilde{a}_{x, y}\right\}$, where 


$$
\tilde{a}_{x, y}=a_{x, y} \exp \left[\frac{2 \pi i x y}{L+1}\right] .
$$

If we use periodic boundary conditions in the y-direction, and $L=M-1$, we can define another toppling invariant $I_{2}$

$$
I_{2}(\mathcal{Z})=\sum_{x, y} x z_{x, y}, \quad \bmod M
$$

Thus, for a lattice of size $L \times(L+1)$, we have two independent toppling invariants $I_{1}$ and $I_{2}$. More generally, if $M \neq L+1$, and the greatest common divisor of $M$ and $L+1$ is $R$, then both $I_{1}$ and $I_{2}$ are invariant modulo $R$.

Using these symmtries together, we can construct a set of $M^{2}$ solutions of the coupled eigenvalue equations (9) when $M=L+1$, for any solution $\left\{a_{x, y}\right\}$. These solutions are parameterized by two integers $k$ and $r$. It is easily checked that if $\left\{a_{x, y}\right\}$ is a solution of Eqs.(9), then so is $\left\{\tilde{a}_{x, y}\right\}$, where

$$
\tilde{a}_{x, y}=a_{x, y} \exp [2 \pi i(k+r y) x / M], \quad 0 \leq k<M, 0 \leq r<M
$$

We can group configuration having the same values of $I_{1}, I_{2}$ into same equivalence class. There are $M^{2}$ such equivalence classes. Clearly, no value of $I_{1}$ or $I_{2}$ has any preferred status, and so, in the steady state, each of these equivalence classes must have equal weight. Thus, the toppling invariants provide a partial characterization of the probabilities of different configuration in the steady state. Also, it is easy to see that all configuration obtained by adding a particle at $(x, y)$ to a configuration belongs to the equivalence class of $I_{1}=i, I_{2}=j$, would belong to the equivalence class with $I_{1}=i+x y, I_{2}=j+x$.

\section{$5 \quad$ Minimal Forbidden Subconfigurations}

Determining the probabilities of different stable configurations in the steady state is not easy. However, as a first step towards such a characterization, we show below that most of the $2^{L M}$ stable configurations do not occur at all in the steady state, and the number of recurrent configurations of the sandpile is only an exponentially small fraction of all stable configurations. We do this by showing that there are many local constraints on the allowed heights $\left\{z_{x, y}\right\}$ in recurrent configurations.

The simplest of such constraints is that in any recurrent configuration, any four neighboring sites forming a $2 \times 2$ square cannot be all unoccupied. Thus the local structure shown in Fig. 1a cannot be found anywhere in a recurrent configuration. We shall call such structures forbidden subconfigurations (FSC's). In fact, we shall show that an FSC cannot even in an intermediate unstable configuration, if we start with a recurrent configuration.

The proof that Fig. 1a is an FSC is similar to the proof used in the DASM case [13]: We start with an initial configuration of the sandpile in which there is no such subconfiguration. Now we evolve the sandpile by adding particles at sites, and toppling any sites which become unstable. We would like to prove that at no stage, in the intervening stable or unstable configurations, would we be able to generate the subconfiguration shown in Fig. 1a, whatever 


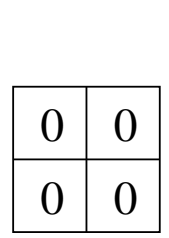

(a)

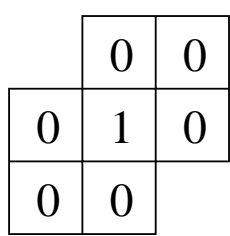

(b)

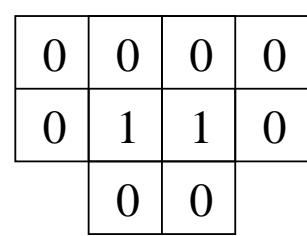

(c)

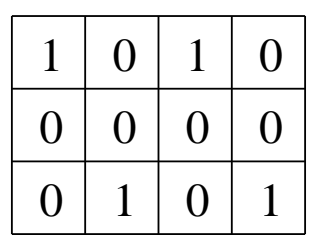

(d)

\begin{tabular}{|l|l|l|l|l|}
\hline 0 & 0 & 0 & 0 & \multicolumn{1}{|c}{} \\
\cline { 1 - 4 } 0 & 1 & 1 & 1 & 0 \\
\hline & 0 & 0 & 0 & 0 \\
\cline { 2 - 4 } & &
\end{tabular}

(e)

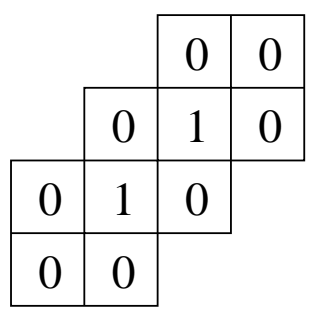

(f)

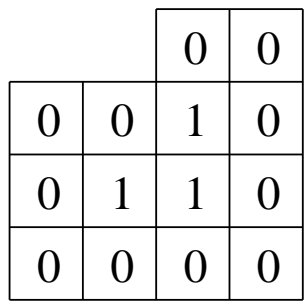

$(\mathrm{g})$

Figure 1: Some minimal forbidden subconfigurations of the Manna Model

the sequence of particle additions, and whatever choice we make for each toppling (horizontal or vertical transfer).

To prove, we assume the contrary. Let us treat each particle addition or toppling as a seperate relaxation step. Let the FSC appears for the first time after the $T$-th step. Then, after step $T-1$, there was no FSC at these sites, and the height at one of these sites must have decreased in the $T$-th step. This implies that the $T$-th step was a toppling at one of the four sites in the $2 \times 2$ square. But this toppling, whether transfer was horizontal or vertical, would send one particle to one of the neighbors in the square. Since the minimum height at the neighbor is zero at time $T-1$, its height at the $T$-th step must be at least 1 , which contradicts the assumption that the height is 0 at all the four sites at the $T$-th step. Hence, no such $T$ can be found, and the assumption is not possible.

We can prove that other configurations shown in Fig. 1 are also FSC's similarly. For example, to show that subconfiguration shown in Fig. 1b is an FSC, we again note that if this subconfiguration is generated for the first time at the $T$-th step, then the previous step must be a toppling at one of the sites in the FSC. That implies that at the $(T-1)$-th step, the height at one of the neighboring sites was negative, or that the central site was of height zero . But the latter case would lead to an configuration shown Fig. 1a, already proved to be a FSC.

If a subconfiguration cannot be generated on a small lattice, it cannot be generated on a bigger lattice either, as the effect of the rest of the lattice on a small part can always be reproduced by particles added at the boundaries of the of the part at appropriate points in space and time. Thus, every transient configuration of a small lattice is an FSC on a bigger lattice.

Clearly, if $\mathbf{F}$ is an FSC, so is any bigger set containing $\mathbf{F}$ as a subset. We may therefore, without any loss, restrict our discussion to FSC's such that any proper subset of the sites 
does not form an FSC. Such FSC's will be called minimal FSC's.

To form a list of all minimal FSC's, one can start by examining the transient configurations on small lattices. It is easily seen that all configurations are recurrent if $M$ or $L=1$, and that Fig. 1a gives the only transient configuration on a $2 \times 2$ lattice. One can check that going to lattices $2 \times M$ with $M>2$ does not generate any new minimal FSC. We then enumerate all the transient configurations of the $3 \times 3$ lattice. Some of these are disallowed by the existence of FSC's of smaller sizes. From the remaining transient configurations, we can get a reduced set of minimal FSC's by replacing the FSC by a smaller set with fewer sites wherever possible. This gives us a new minimal FSC shown in Fig. 1b ( and another FSC related to this by reflection symmetry). Going to bigger lattices, we can continue to augment our list of FSC's, with larger and larger FSC's. At each stage, we can be sure that all minimal FSC's having fewer than a specific number of sites have been enumerated. Some of the minimal FSC's constructed in this way are shown in Fig. 1 b-g. If we confined our attention to FSC's on stable configurations only, and not insist that they be forbidden in unstable states also, these configurations could have been made smaller by omitting all sites of height 1 in the figure.

The existence of FSC's implies local inequality contraints in the height variables that must be satisfied at all sites in recurrent states. This implies that the fraction of the $2^{L M}$ stable configurations that are recurrent must decrease to zero for large lattices (exponentially fast in the number of sites in the lattice).

The procedure to construct FSC's is rather tedious, and not very illuminating as the number of minimal FSC's is infinite. It seems desirable to have a simple algorithm which can identify FSC's. For the DASM, such an algorithm is rather simple: it is known as the burning test [15], or the script test for some asymmetric graphs [14].

A simple variation of the burning test seems to work well for a large majority of configurations: starting with the given configuration, one recursively burns any site $i$ for which the height $z_{i} \geq \operatorname{Min}\left(v_{i}, h_{i}\right)$, where $v_{i}$ and $h_{i}$ are the number of vertical and horizontal unburnt neighbors of $i$ respectively. If eventually all sites are burnt, the configuration passes this burning test, else not.

Some not-very-systematic checking on small lattices showed that if a configuration passes this modified burning test, it is usually recurrent, and if it fails, it is transient. However, there are a few instances where this conclusion is incorrect. For example, the FSC shown in Fig. 1d burns completely, and hence should have been recurrent according to this test. One can also find configurations that do not burn according to this test, but are recurrent. An example is given in Fig. 2. It is possible that a small change in the burning condition in this test would be make it work without any error. Finding such a refinement remains an open problem at present.

We note that the existence of FSC's is a special feature of these toppling rules. If we had chosen the particle transfer to be in the north-and-east or south-and-west directions with equal probability, then it is easy to see that starting from any configuration, we can get to the configuration with all sites empty, and hence all stable configurations are recurrent. 


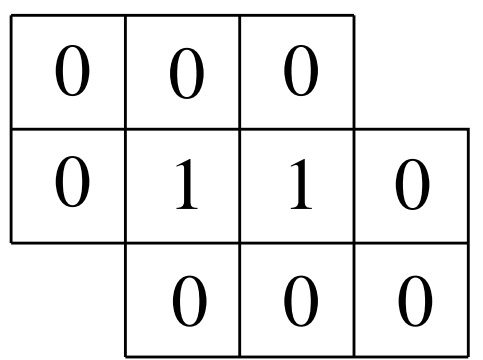

Figure 2: A subconfiguration that does not burn in the modied burning test, but is not an FSC.

\section{Minimal Sand Configurations}

Let $\mathcal{R}$ be a recurrent configuration of sandpile on a rectangle of size $\ell \times m$. We denote the number of sandgrains in $\mathcal{R}$ by $N(\mathcal{R})$. The minimum value of $N(\mathcal{R})$ over all recurrent configurations on the lattice will be denoted by $N_{\min }(\ell, m)$. In this section we study how $N_{\text {min }}(\ell, m)$ depends on $\ell$ and $m$. For large $\ell, m$, this should vary linearly with the number of sites in the lattice, and we define the minimum possible density of occupied sites in a recurrent configuration as $\rho_{\text {min }}$. Clearly,

$$
\rho_{\text {min }}=\operatorname{Lim}_{\ell, m \rightarrow \infty} \frac{N_{\min }(\ell, m)}{\ell m} .
$$

We have already seen that four adjacent sites forming a $2 \times 2$ square, if all unoccupied, form an FSC, and hence each such group of four sites must have at least one occupied site. For large $\ell, m$, we can divide the space into non-overlapping $2 \times 2$ squares, and so we see that

$$
\rho_{\min } \geq \frac{1}{4}
$$

One can get better bounds on $\rho_{\min }$ using bigger tiling units. In Fig. 3, we have shown the tilings using tiles using 7 and 12 sites each. For the 7 -site tile, it is easy to see that we get an FSC within the tile, unless the number of occupied sites within the tile is at least 2 . This implies that $\rho_{\min } \geq \frac{2}{7}$. For the $12-$ sited tiling, the minimum number of occupied site per tile can easily be checked to be 4 . This gives us an improved bound $\rho_{\min } \geq \frac{1}{3}$.

It is straight-forward to extend this procedure to larger tiles. For any finite tile, there are only a finite number of configurations, and by exhaustive enumeration, one can find the largest number $r$, such that all configurations with less than $r$ occupied sites necessarily contain an FSC, and are transient. The number of different configurations to be checked increases as $2^{\ell m}$, and this brute-force approach does not seem to be very practical for large $\ell, m$. However, experimenting with rectangular tiles of size $\ell \times m$ leads us to conjecture that

$$
N_{\min }(\ell, m)=f(\ell, m)
$$



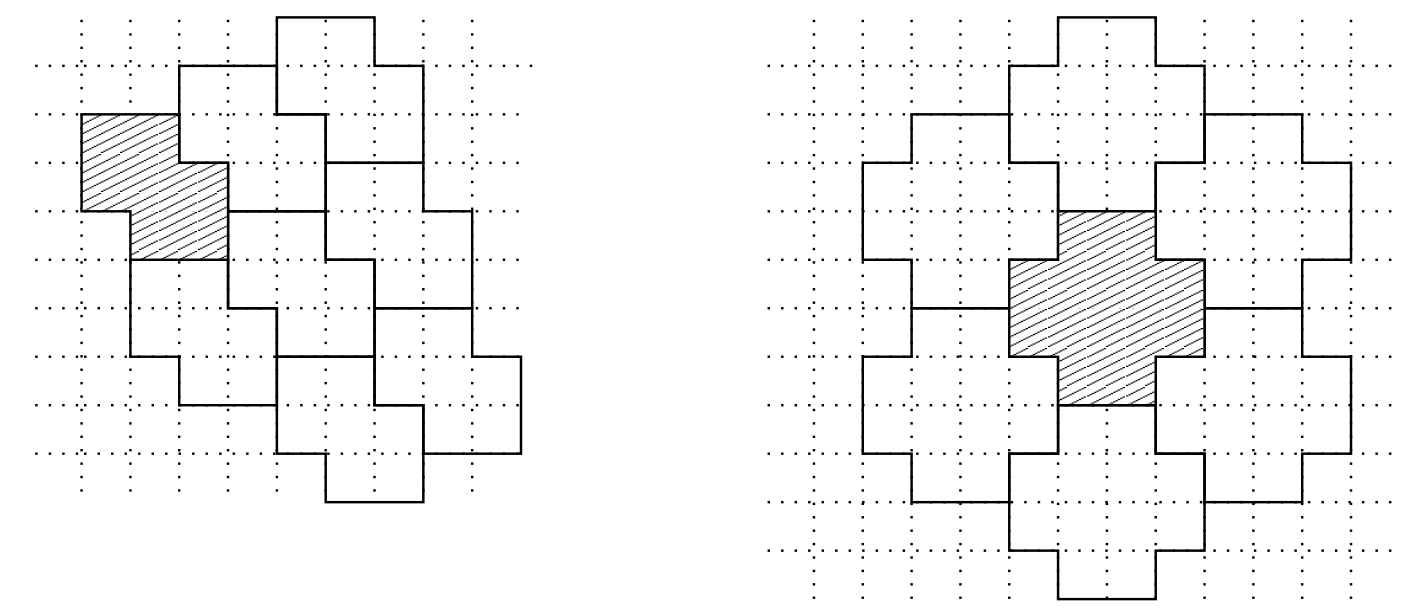

Figure 3: Tiling of the plane using tiles of 7 and 12 sites.

where

$$
\begin{aligned}
f(\ell, m) & =\frac{1}{2}(\ell-1)(m-1), \quad \text { if } \ell \text { or } m \text { odd } \\
& =\frac{1}{2}(\ell m-\ell-m+2), \quad \text { otherwise }
\end{aligned}
$$

We have checked that the conjecture is true for $1 \leq \ell, m \leq 4$, and if $\ell \leq 2$ for all $m$. Note that in the limit of large $\ell, m$, this implies that

$$
\rho_{\min }=\frac{1}{2}
$$

As additional support for this conjecture, we show that $N_{\min }(\ell, m)$ cannot exceed $f(\ell, m)$. This is done by explicitly constructing a recurrent configuration having $f(\ell, m)$ particles. We recall the definition that a configuration is recurrent if it occurs with non-zero probability in the steady state of the system. The configuration with all sites occupied can clearly be reached from any configuration of the system ( by adding particles only at unoccupied sites), and is recurrent. Thus a configuration is recurrent if and only if there is a non-zero probability that it is reached in a finite number of steps from the fully occupied configuration.

We now show that for all values of $\ell$ and $m$, starting from a fully occupied configuration of size $\ell \times m$, we can construct a sequence of topplings and particle additions that makes the sandpile reach a configuration in which the number of particles is $f(\ell, m)$. The claim is easily checked for $\ell, m \leq 2$. For larger values of $\ell, m$, we prove this result by induction. Assume that The claim is true for some value $\left(\ell_{0}, m_{0}\right)$. We now show that it must then also be true for $\left(\ell=\ell_{0}+2, m_{0}\right)$.

We construct the minimal configuration from the fully occupied configuration of the $\left(\ell_{0}+2, m_{0}\right)$ lattice as follows: In the initial configuration, $\left\{z_{x, y}\right\}=1$, for all $x, y$. We first produce a configuration on the rectangle $0 \leq x \leq \ell_{0}, 0 \leq y \leq m_{0}$ having only $f\left(\ell_{0}, m_{0}\right)$ sandgrains. This can be done, by assumption. In this process, topplings at the column 
$x=\ell_{0}$ would add particles to the column $x=\ell_{0}+1$. We do not relax these yet, and add a particle to each site of the column $x=\ell_{0}+2$, and topple it horizontally. Finally, we relax the sites in the column $x=\ell_{0}+1$ by toppling them in the vertical direction only. In the $x=\ell_{0}+1$ column, we essentially have a one dimensional ASM with deterministic topplings. Thus, there can be at most one unoccupied site in the column after relaxation. [If all sites in the column are occupied, one vacancy is created on adding one more particle at any site in the column, and relaxing, again transferring particles only in the vertical direction]. The final number of particles in the configuration is clearly $f\left(\ell_{0}, m_{0}\right)+(m-1)=f\left(\ell_{0}+2, m_{0}\right)$.

By symmetry between the horizontal and vertical directions, we have $N_{\min }(\ell, m)=$ $N_{\min }(m, \ell)$. Hence, by induction on $\ell$ and $m$, we conclude that

$$
N_{\min }(\ell, m) \leq f(\ell, m), \quad \text { for all } \ell, m \text {. }
$$

where $f(\ell, m)$ is the integer function defined by eq(20). A proof of the conjectured equality eq.(18) remains an open problem.

I thank Satya N. Majumdar for a critical reading of the manuscript.

\section{References}

[1] Bak P., Tang C. and Wiesenfeld K., Phys. Rev. Lett., 59(1987) 381.

[2] Bak P., Tang C. and Wiesenfeld K., Phys. Rev.,A 38(1988) 364.

[3] Dhar D., Physica A 263 (1999) 4.

[4] Kadanoff L. P., Nagel S. R., Wu L. and Zhou S., Phys. Rev. A 39 (1989) 6524.

[5] Manna S. S., Physica A 179 (1991) 249.

[6] Zhang Y.-C., Phys. Rev. Lett., 63 (1989) 470.

[7] Dhar D. and Ramaswamy R., Phys. Rev. Lett. 63 (1989) 1659.

[8] Manna S. S., J. Phys A 24 (1991) L363.

[9] Tadic B. and Dhar D., Phys. Rev. Lett. 79 (1997) 1519.

[10] Ben-Hur A. and Biham O., Phys. Rev. E 53 (1996) R1317.

[11] Frette V., Christensen K., Malthe-Sorensen, Feder J., Josang T. and Meakin P., Nature 379 (1996) 49.

[12] Dhar D., Ruelle P., Sen S. and Verma D.-N., J, Phys. A 28 (1995) 805.

[13] Dhar D., Phys. Rev. Lett. 67 (1990) 1613.

[14] Speer E. R., J. Stat. Phys. 71 (1993) 61.

[15] Majumdar S. N. and Dhar D., Physica A 185 (1992) 129. 